\title{
Uma Técnica de Múltiplo Acesso Baseada na Auto-estrutura das Transformadas Trigonométricas
}

\author{
J. B. Lima e R. M. Campello de Souza
}

\begin{abstract}
Resumo-Este artigo propõe uma técnica de múltiplo acesso ao canal somador real (RAC). Neste cenário, a separação da informação enviada pelos usuários baseia-se no fato de que, a cada um deles, está associado um conjunto específico de auto-sequiências de uma transformada trigonométrica discreta (DTT). Um estudo acerca da auto-estrutura das matrizes de transformação das DTTs é apresentado. Particularmente, analisase os autovalores e os autovetores da transformada discreta do cosseno (DCT).
\end{abstract}

Palavras-Chave-Múltiplo acesso, canal somador real, transformadas trigonométricas discretas, auto-seqüiências.

Abstract-This paper proposes a multiple access technique for the real adder channel (RAC). In this framework, the separation of the information sent by the users is based on the fact that a specific set of eigensequences of a discrete trigonometric transform (DTT) is associated to each of them. Results concerning the eigenstructure of the DTTs transformation matrices are presented. Particularly, the discrete cosine transform (DCT) eigenvalues and eigenvectors are analyzed.

Keywords-Multiple access, real adder channel, discrete trigonometric transforms, eigensequences.

\section{INTRODUÇÃO}

Em Processamento de Sinais, as transformadas trigonométricas discretas (DTTs, discrete trigonometric transforms) desempenham um relevante papel, fundamentando uma série de técnicas presentes em diversas aplicações. $\mathrm{Na}$ área de Processamento Digital de Imagem, particularmente, a transformada discreta do cosseno (DCT, discrete cosine transform) é uma das principais ferramentas, constituindo a base para importantes padrões de compressão de sinais e para técnicas de marca d'água no domínio da freqüência [1], [2].

$\mathrm{Na}$ maioria dos casos, o que torna atrativo o uso da DCT é a sua propriedade de compactação de energia. A definição de cada tipo de DTT pode ser feita a partir de uma extensão simétrica da seqüência cuja transformada se deseja calcular [3]. No caso da DCT, realiza-se um tipo de extensão que proporciona uma suavização do sinal, o que resulta na concentração da energia do mesmo nas componentes da transformada associadas às freqüências mais baixas.

Aplicações que focalizam diferentes propriedades da DCT e de outras transformadas trigonométricas têm, contudo, sido desenvolvidas. Em um trabalho recente, com base na convolução simétrica das DTTs, propôs-se o seu emprego em sistemas OFDM (Orthogonal Frequency Division Multiplexing) [4]; mostra-se, também, que as transformadas discretas fracionais

Juliano Bandeira Lima e Ricardo M. Campello de Souza, Grupo de Processamento de Sinais, Departamento de Eletrônica e Sistemas, Universidade Federal de Pernambuco, Recife, Brasil, E-mails: juliano_bandeira@ieee.org, ricardo@ufpe.br. do cosseno e do seno podem ser bastante úteis no campo da óptica [5]; noutros casos, essas ferramentas têm sido integradas a algoritmos rápidos para a solução de sistemas Toeplitz [6].

O objetivo deste artigo é propor uma técnica de múltiplo acesso ao canal somador real (RAC), baseada na estrutura de autovetores e autovalores (auto-estrutura) das DTTs. A auto-estrutura das transformadas discretas foi investigada pela primeira vez por Parks e McClellan, que consideraram a transformada discreta de Fourier (DFT, discrete Fourier transform) [7]. No referido trabalho, foram determinadas as formas dos autovetores da DFT e as multiplicidades dos seus respectivos autovalores. Posteriormente, outros trabalhos realizaram uma análise semelhante, considerando alguns tipos de transformadas trigonométricas [5], [8], [9].

Após esta introdução, são brevemente revistas as definições das 16 transformadas pertencentes à família das DTTs. Na seção III, a auto-estrutura das transformadas trigonométricas é discutida. Na seção IV, é proposta uma aplicação desses conceitos, um método de acesso múltiplo ao canal somador real. A técnica consiste, basicamente, em utilizar auto-seqüências que podem ser "misturadas" num mesmo canal e, posteriormente, recuperadas. Finalmente, algumas conclusões e perspectivas para futuras investigações são apresentadas.

\section{TRANSFORMADAS TRIGONOMÉTRICAS DISCRETAS}

Uma das maneiras de construir as transformadas trigonométricas é considerar uma seqüência $x=\left(x_{i}\right)$ de comprimento $N, N-1$ ou $N+1$, e estendê-la para a esquerda e para a direita segundo determinado critério de simetria. Combinando uma simetria par ou ímpar, com uma simetria cujo eixo se localiza sobre uma amostra ou sobre o ponto médio entre duas amostras, é possível criar 4 tipos de simetria (Figura 1): WS (whole-sample symmetry), WA (whole-sample antisymmetry), HS (half-sample symmetry) e HA (half-sample antisymmetry). O procedimento de extensão de $x$ gera uma nova sequiência $\hat{x}=\left(\hat{x}_{i}\right)$. Os coeficientes da respectiva transformada trigonométrica de $x$ são obtidos a partir da DFT de um trecho de comprimento $2 N$ ou $2 N-1$ de $\hat{x}$. Isso significa que é possível definir 16 versões de DTTs usando senos e cossenos [3].

De maneira geral, qualquer DTT de uma seqüência ou vetor linha $x=\left(x_{i}\right)$ é um vetor coluna $X=\left(X_{k}\right)$, que se obtém pela equação matricial

$$
X=M x^{T},
$$

onde $M$ é a matriz de transformação. Os elementos dessa matriz são computados de acordo com as tabelas I e II, as 


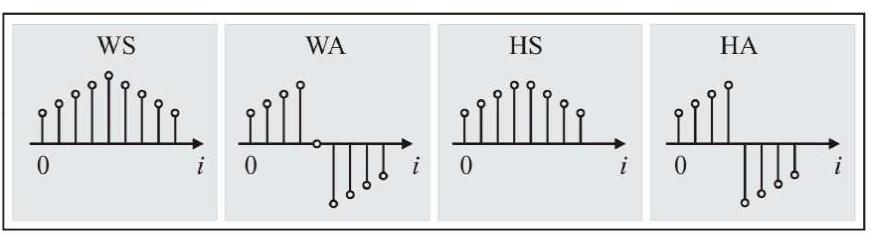

Fig. 1. Tipos de simetria de uma seqüência.

quais, resumidamente, apresentam cada DTT em sua versão unitária. Para simplificar, as transformadas do cosseno e as do seno são denotadas por $\mathcal{C}$ e $\mathcal{S}$, respectivamente. Na segunda coluna de cada tabela, observa-se a forma como variam os índices $k$ (linhas) e $i$ (colunas), que determinam as dimensões de $x, M$ e $X$. As funções peso são dadas por

$$
\beta_{r}= \begin{cases}\frac{1}{\sqrt{2}}, & r=0 \text { ou } N \\ 1, & r=1,2, \ldots, N-1\end{cases}
$$

e

$$
\gamma_{r}= \begin{cases}1, & r=0,1, \ldots, N-2, \\ \frac{1}{\sqrt{2}}, & r=N-1 .\end{cases}
$$

Naturalmente, o cálculo da DTT inversa de uma seqüência também pode ser representado através de uma equação matricial. As relações entre cada transformada e sua inversa são dadas pelas equações

$$
\begin{aligned}
& {\left[\mathcal{C}_{1}\right]^{-1}=\left[\mathcal{C}_{1}\right]} \\
& {\left[\mathcal{C}_{2}\right]^{-1}=\left[\mathcal{C}_{3}\right]} \\
& {\left[\mathcal{C}_{3}\right]^{-1}=\left[\mathcal{C}_{2}\right]} \\
& {\left[\mathcal{C}_{4}\right]^{-1}=\left[\mathcal{C}_{4}\right]} \\
& {\left[\mathcal{S}_{1}\right]^{-1}=\left[\mathcal{S}_{1}\right]} \\
& {\left[\mathcal{S}_{2}\right]^{-1}=\left[\mathcal{S}_{3}\right]} \\
& {\left[\mathcal{S}_{3}\right]^{-1}=\left[\mathcal{S}_{2}\right]} \\
& {\left[\mathcal{S}_{4}\right]^{-1}=\left[\mathcal{S}_{4}\right] .}
\end{aligned}
$$

\section{Auto-Estrutura DAS DTTS}

O objetivo central desta seção é determinar que seqüências $x_{i}$ possuem uma transformada trigonométrica $X_{k}$ que satisfaz a relação

$$
X_{k}=\lambda x_{k}
$$

A Equação (12) pode ser escrita sob a forma $\mathcal{T}\left\{x_{i}\right\}=$ $\lambda x_{i}$, onde $\mathcal{T}$ denota o operador transformada trigonométrica discreta, $\lambda$ representa um autovalor do mesmo e $x_{i}$ o seu autovetor (ou auto-sequiência) associado. Assim, para determinar as auto-seqüências de uma DTT específica, é suficiente obter os autovalores de sua matriz de transformação. Basicamente, a auto-estrutura dessas transformadas se divide em dois casos, que são analisados em seguida.

\section{A. Autovalores das DTTs dos tipos 1 e 4 .}

Os autovalores associados a uma transformada discreta possuem uma relação direta com o que se chama de ciclo da matriz de transformação. Este parâmetro pode ser entendido como o número de vezes sucessivas que se deve calcular a transformada de uma sequiência até que se obtenha como
TABELA I

TRANSFORMADAS DISCRETAS DO COSSENO.

\begin{tabular}{|l|l|}
\hline Elementos da matriz de transformação & Dimensão da matriz \\
\hline \hline$\left[\mathcal{C}_{1 e}\right]_{k i}=\sqrt{\frac{2}{N}} \beta_{i} \beta_{k} \cos \left(\frac{\pi k i}{N}\right)$ & $0 \leq k, i \leq N$ \\
\hline$\left[\mathcal{C}_{2 e}\right]_{k i}=\sqrt{\frac{2}{N}} \beta_{k} \cos \left(\frac{\pi k\left(i+\frac{1}{2}\right)}{N}\right)$ & $0 \leq k, i \leq N-1$ \\
\hline$\left[\mathcal{C}_{3 e}\right]_{k i}=\sqrt{\frac{2}{N}} \beta_{i} \cos \left(\frac{\pi\left(k+\frac{1}{2}\right) i}{N}\right)$ & $0 \leq k, i \leq N-1$ \\
\hline$\left[\mathcal{C}_{4 e}\right]_{k i}=\sqrt{\frac{2}{N}} \cos \left(\frac{\pi\left(k+\frac{1}{2}\right)\left(i+\frac{1}{2}\right)}{N}\right)$ & $0 \leq k, i \leq N-1$ \\
\hline$\left[\mathcal{C}_{1 o}\right]_{k i}=\frac{2}{\sqrt{2 N-1}} \beta_{i} \beta_{k} \cos \left(\frac{2 \pi k i}{2 N-1}\right)$ & $0 \leq k, i \leq N$ \\
\hline$\left[\mathcal{C}_{2 o}\right]_{k i}=\frac{2}{\sqrt{2 N-1}} \gamma_{i} \beta_{k} \cos \left(\frac{2 \pi k\left(i+\frac{1}{2}\right)}{2 N-1}\right)$ & $0 \leq k, i \leq N-1$ \\
\hline$\left[\mathcal{C}_{3 o}\right]_{k i}=\frac{2}{\sqrt{2 N-1}} \beta_{i} \gamma_{k} \cos \left(\frac{2 \pi\left(k+\frac{1}{2}\right) i}{2 N-1}\right)$ & $0 \leq k, i \leq N-1$ \\
\hline$\left[\mathcal{C}_{4 o}\right]_{k i}=\frac{2}{\sqrt{2 N-1}} \cos \left(\frac{2 \pi\left(k+\frac{1}{2}\right)\left(i+\frac{1}{2}\right)}{2 N-1}\right)$ & $0 \leq k, i \leq N-2$ \\
\hline
\end{tabular}

TABELA II

TRANSFORMADAS DISCRETAS DO SENO.

\begin{tabular}{|l|l|}
\hline Elementos da matriz de transformação & Dimensão da matriz \\
\hline \hline$\left[\mathcal{S}_{1 e}\right]_{k i}=\sqrt{\frac{2}{N}} \sin \left(\frac{\pi k i}{N}\right)$ & $1 \leq k, i \leq N-1$ \\
\hline$\left[\mathcal{S}_{2 e}\right]_{k i}=\sqrt{\frac{2}{N}} \beta_{k} \sin \left(\frac{\pi k\left(i+\frac{1}{2}\right)}{N}\right)$ & $1 \leq k \leq N$ \\
\hline$\left[\mathcal{S}_{3 e}\right]_{k i}=\sqrt{\frac{2}{N}} \beta_{i} \sin \left(\frac{\pi\left(k+\frac{1}{2}\right) i}{N}\right)$ & $0 \leq i \leq N-1$ \\
\hline$\left[\mathcal{S}_{4 e}\right]_{k i}=\sqrt{\frac{2}{N}} \sin \left(\frac{\pi\left(k+\frac{1}{2}\right)\left(i+\frac{1}{2}\right)}{N}\right)$ & $0 \leq k \leq N-1$ \\
\hline$\left[\mathcal{S}_{1 o}\right]_{k i}=\frac{2}{\sqrt{2 N-1}} \sin \left(\frac{2 \pi k i}{2 N-1}\right)$ & $1 \leq i \leq N$ \\
\hline$\left[\mathcal{S}_{2 o}\right]_{k i}=\frac{2}{\sqrt{2 N-1}} \sin \left(\frac{2 \pi k\left(i+\frac{1}{2}\right)}{2 N-1}\right)$ & $1 \leq k, i \leq N-1$ \\
\hline$\left[\mathcal{S}_{3 o}\right]_{k i}=\frac{2}{\sqrt{2 N-1}} \sin \left(\frac{2 \pi\left(k+\frac{1}{2}\right) i}{2 N-1}\right)$ & $1 \leq k \leq N-1$ \\
\hline$\frac{2}{\sqrt{2 N-1}} \gamma_{i} \gamma_{k} \sin \left(\frac{2 \pi\left(k+\frac{1}{2}\right)\left(i+\frac{1}{2}\right)}{2 N-1}\right)$ & $0 \leq k, i \leq N-1$ \\
\hline
\end{tabular}

resultado a mesma seqüência. Tal procedimento é equivalente a obter a ordem multiplicativa da matriz, isto é, o menor 
expoente positivo ao qual a mesma, sendo elevada, resulta na matriz identidade. Esse fato é ilustrado a partir da proposição a seguir.

Proposição 1: Os autovalores da matriz $\left[\mathcal{C}_{1 e}\right]$ são $\lambda=1 \mathrm{e}$ $\lambda=-1$.

Demonstração: Observando a Equação (4), verifica-se que $\mathcal{C}_{1 e}$ é um operador involucionário, ou seja, o seu ciclo é igual a 2. Dessa forma, aplicando-o duas vezes sucessivas a uma seqüência $x_{i}$, obtém-se os pares

$$
\begin{aligned}
x_{i} \leftrightarrow X_{k}, \\
X_{i} \leftrightarrow x_{k} .
\end{aligned}
$$

Equivalentemente, pode-se escrever

$$
\mathcal{C}_{1 e}^{2}\left\{x_{i}\right\}=x_{k}
$$

Como $x_{i}$ é uma auto-seqüência de $\mathcal{C}_{1 e}$, a última expressão corresponde a $\lambda^{2} x_{k}=x_{k}$, de onde se chega ao resultado proposto.

A Proposição 1 pode ser estendida às demais transformadas trigonométricas dos tipos 1 e 4, sejam as mesmas do cosseno ou do seno; com simetria par ou ímpar. Isso porque esses operadores também possuem ciclo igual a 2 , o que, conforme demonstrado, é suficiente para se obter os seus autovalores.

\section{B. Autovalores das DTTs dos tipos 2 e 3 .}

A obtenção dos autovalores das DTTs dos tipos 2 e 3 requer uma abordagem diferente da apresentada na Proposição 1, uma vez que as mesmas não são involuções. Dessa maneira, recorrer-se-á ao procedimeto convencional para o cálculo dos autovalores de uma matriz, que corresponde à avaliação das raízes do polinômio característico da mesma. Para isso, será considerada a matriz $\left[\mathcal{C}_{2 e}\right]$. As outras DTTs, dos tipos 2 e 3 , apresentarão um comportamento semelhante.

Como a determinação do polinômio característico de uma matriz envolve o cálculo de um determinante, é relevante mencionar que o custo computacional desta tarefa, normalmente alto, pode ser diminuído. Isso é feito, por exemplo, reduzindo a matriz à sua forma de Hessenberg ou tridiagonal [11]. Além disso, a ortogonalidade das matrizes das DTTs, verificada diretamente a partir das expressões que as definem, permite afirmar que seus autovalores possuirão módulo igual a 1 e que seus polinômios apresentarão um tipo especial de simetria.

Teorema 1: O polinômio característico de uma matriz ortogonal é um polinômio recíproco.

Demonstração: Seja $M$ uma matriz ortogonal com dimensões $N \times N$ e $p(\lambda)=\operatorname{det}(M-\lambda I)$ o seu polinômio característico. Deseja-se provar que

$$
p(\lambda)= \pm \lambda^{N} p(1 / \lambda) .
$$

Como $M^{-1}=M^{T}$, tem-se $M-\lambda I=-\lambda M\left(M^{T}-I / \lambda\right)$. Considerando o determinante em ambos os lados da última expressão e usando os fatos de que $\operatorname{det}(M)=\operatorname{det}\left(M^{T}\right)= \pm 1$ e $\operatorname{det}(\lambda M)=\lambda^{N} \operatorname{det}(M), \lambda \in \mathbb{C}$, tem-se

$$
\operatorname{det}(M-\lambda I)= \pm \lambda^{N} \operatorname{det}\left(M-\frac{1}{\lambda} I\right) .
$$

Os polinômios em questão são denominados palindrômicos, caso $p(\lambda)=\lambda^{N} p(1 / \lambda)$, ou anti-palindrômicos, caso $p(\lambda)=$ $-\lambda^{N} p(1 / \lambda)$. O cálculo das raízes de polinômios desse tipo é facilitado por conta de um método de substituição de variáveis que reduz o seu grau à metade [12]. Assim, é possível utilizar fórmulas fechadas para avaliar os zeros de um polinômio palindrômico de grau até 10 . Retirando-se as raízes 1 e -1 , o mesmo é reduzido a um polinômio de grau 4. Acima disso, métodos numéricos podem ser empregados.

Com o auxílio de programas de computador, obteve-se os polinômios característicos das matrizes de transformação da $\mathcal{C}_{2 e}$ de diversos comprimentos e suas respectivas raízes. Os resultados observados permitem conjecturar que, para qualquer comprimento $N$, os autovalores da matriz $\left[\mathcal{C}_{2 e}\right]$ são todos distintos.

Outro importante aspecto dessa matriz pode ser percebido escrevendo-a na sua forma diagonal, como a seguir,

$$
\left[\mathcal{C}_{2 e}\right]=\mathbf{U} \boldsymbol{\Lambda} \mathbf{U}^{*} .
$$

Na última equação, U é uma matriz unitária cujas colunas são autovetores da matriz $\left[\mathcal{C}_{2 e}\right]$ (U* é sua transposta conjugada); $\boldsymbol{\Lambda}$ é uma matriz diagonal cujos elementos são os autovalores de $\left[\mathcal{C}_{2 e}\right]$. Conforme será demonstrado, essa representação auxilia o estudo do ciclo da matriz de transformação.

Diretamente da Equação (16), observa-se que potências de $\left[\mathcal{C}_{2 e}\right]$ podem ser calculadas a partir de potências de $\boldsymbol{\Lambda}$, pois, uma vez que $\mathbf{U}^{*} \mathbf{U}=\mathbf{I}$,

$$
\left[\mathcal{C}_{2 e}\right]^{r}=\mathbf{U} \boldsymbol{\Lambda}^{r} \mathbf{U}^{*}
$$

onde $r$ é um número inteiro. Assim, o menor valor positivo de $r$ que fornece $\left[\mathcal{C}_{2 e}\right]^{r}=\mathbf{I}$ é o mesmo que fornece $\boldsymbol{\Lambda}^{r}=\mathbf{I}$. Como $\boldsymbol{\Lambda}$ é diagonal, o cálculo de suas potências é feito simplesmente elevando cada componente ao respectivo expoente. No presente caso, tais componentes são os autovalores de $\left[\mathcal{C}_{2 e}\right]$ que, sendo unimodulares, podem ser escritos como $\lambda_{i}=e^{j \theta_{i}}$, $i=0,1, \ldots, N-1$. O valor de $r$ que se busca é, portanto, aquele que produz $r \theta \equiv 0(\bmod 2 \pi)$, simultaneamente para todo $i$. Para isso, seria necessário que todos os argumentos $\theta_{i}$ fossem frações racionais de $2 \pi$, o que se conjectura que não acontece para a matriz $\left[\mathcal{C}_{2 e}\right]$. Esse fato foi observado através de simulações computacionais em que potências de $\left[\mathcal{C}_{2 e}\right]$ de diversos valores foram calculadas. Por definição, o ciclo de tal matriz seria 0 .

\section{Múltiplo Acesso Via Transformadas TRIGONOMÉTRICAS}

O canal somador binário com dois usuários (2-BAC, twouser binary adder channel) é um modelo de canal de comunicação bastante conhecido [10]. Em [13], foi proposto um modelo de canal somador real (RAC, real adder channel) onde, pela primeira vez, auto-seqüências de uma transformada discreta, a de Fourier, foram utilizadas para prover o acesso simultâneo de até 4 usuários.

Nesta seção, mostra-se como a idéia de múltiplo acesso ao canal somador real pode ser implementada utilizando autoseqüências de transformadas trigonométricas. Nesse caso, o aspecto de maior relevância é o fato de que DTTs dos 
tipos 2 e 3 , de comprimento $N$, possuem $N$ autovalores distintos (conjectura). Isso significa que o número de usuários multiplexados não se limita a 4, que é o número de autovalores distintos da DFT.

\section{A. 2-RAC}

A implementação do 2-RAC baseia-se na auto-estrutura de uma DTT que possui pelo menos 2 autovalores distintos. Como exemplo, considera-se a transformada $\mathcal{C}_{1 e}$ que, conforme demonstrado, possui $\lambda_{1}=1$ e $\lambda_{2}=-1$ como autovalores. Aos usuários 1 e 2 estão associadas as autosequiências $x_{1}=\left(x_{1, i}\right)$ e $x_{2}=\left(x_{2, i}\right)$, relacionadas de modo respectivo aos autovalores mencionados, que podem ser construídas através de regras específicas [8], [13]. Sob esse aspecto, a presente técnica pode ser comparada a uma espécie de DS-CDMA, onde as sequiências de espalhamento seriam os autovetores da matriz de transformação considerada [14].

A partir de

$$
y_{i}=x_{1, i}+x_{2, i},
$$

onde, para simplificar, utiliza-se o sinal "+" para denotar soma componente a componente de seqüências, é possível recuperar as seqüências dos usuários. Calculando $Y_{k}=\mathcal{C}_{1 e}\left\{y_{i}\right\}$, tem-se

$$
Y_{k}=\lambda_{1} x_{1, i}+\lambda_{2} x_{2, i}=x_{1, i}-x_{2, i} .
$$

Das Equações (18) e (19), as seqüências dos usuários são obtidas por

$$
x_{1, i}=\frac{y_{i}+Y_{i}}{2}
$$

$\mathrm{e}$

$$
x_{2, i}=\frac{y_{i}-Y_{i}}{2}
$$

onde $Y$ pode ser calculada usando algoritmos rápidos para a DCT.

\section{B. $4-R A C$}

Considerando a transformada $\mathcal{C}_{2 e}$ de comprimento 4 , a qual estão associados os autovalores

$$
\begin{array}{ll}
\lambda_{1}=0,9904+j 0,1379, & \lambda_{2}=0,9904-j 0,1379, \\
\lambda_{3}=-0,9904+j 0,1379, & \lambda_{4}=-0,9904-j 0,1379,
\end{array}
$$

gera-se um sistema com 4 equações, a fim de recuperar as seqüências dos usuários, $x_{1}=\left(x_{1, i}\right), x_{2}=\left(x_{2, i}\right), x_{3}=$ $\left(x_{3, i}\right)$ e $x_{4}=\left(x_{4, i}\right)$. Assim, ter-se-á

$$
\begin{aligned}
x_{1, i}+x_{2, i}+x_{3, i}+x_{4, i} & =y_{i} \\
\lambda_{1} x_{1, i}+\lambda_{2} x_{2, i}+\lambda_{3} x_{3, i}+\lambda_{4} x_{4, i} & =\mathcal{C}_{2 e}\left\{y_{i}\right\} \\
\lambda_{1}^{2} x_{1, i}+\lambda_{2}^{2} x_{2, i}+\lambda_{3}^{2} x_{3, i}+\lambda_{4}^{2} x_{4, i} & =\mathcal{C}_{2 e}^{2}\left\{y_{i}\right\} \\
\lambda_{1}^{3} x_{1, i}+\lambda_{2}^{3} x_{2, i}+\lambda_{3}^{3} x_{3, i}+\lambda_{4}^{3} x_{4, i} & =\mathcal{C}_{2 e}^{3}\left\{y_{i}\right\}
\end{aligned}
$$

Resolvendo o sistema acima, o que só precisa ser feito uma única vez, é possível expressar $x_{1}, x_{2}, x_{3}$ e $x_{4}$ em função de $y$ e de suas sucessivas transformadas do tipo $\mathcal{C}_{2 e}$. Esse resultado pode ser apresentado através da equação matricial

$$
\mathbf{x}=\left[\begin{array}{cccc}
a & -b & -c & d \\
a^{*} & -b^{*} & c & d^{*} \\
a^{*} & b^{*} & c & -d^{*} \\
a & b & -c & -d
\end{array}\right] \mathbf{y}
$$

onde

$$
\begin{aligned}
& \mathbf{x}=\left[x_{1, i} \ldots x_{4, i}\right]^{T},
\end{aligned}
$$

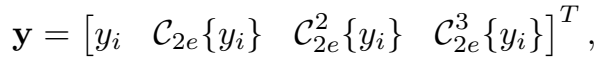

$$
\begin{aligned}
& a=0,2500+j 0,8802, b=0,1262+j 0,9065 \text {, } \\
& c=j 0,9151, d=0,3690+j 0,8374
\end{aligned}
$$

e o símbolo “*” representa o complexo conjugado.

É importante enfatizar que o comprimento das seqüências dos usuários poderia ser maior. Para isso, seria suficiente definir uma transformada $\mathcal{C}_{2 e}$ com comprimento também maior e utilizar apenas 4 dos seus autovalores para conceber o sistema.

\section{8-RAC}

De forma análoga aos exemplos anteriores, a implementação do múltiplo acesso ao 8-RAC deve se basear numa transformada com pelo menos 8 autovalores distintos. Novamente, será considerada a transformada $\mathcal{C}_{2 e}$ que, possuindo comprimento igual a 8 , estará associada aos autovalores

$$
\begin{array}{ll}
\lambda_{1}=0,9592+j 0,2829, & \lambda_{2}=0,9592-j 0,2829, \\
\lambda_{3}=0,9965+j 0,0838, & \lambda_{4}=0,9965+j 0,0838, \\
\lambda_{5}=-0,9604+j 0,2785, & \lambda_{6}=-0,9604-j 0,2785, \\
\lambda_{7}=-0,9969+j 0,0792, & \lambda_{8}=-0,9969-j 0,0792 .
\end{array}
$$

Para $n=1,2, \ldots, 8$, a $n$-ésima equação do sistema cuja solução permite recuperar as seqüências dos usuários, $x_{r}=$ $\left(x_{r, i}\right), \quad(r=1,2, \ldots, 8)$, pode ser expressa por

$$
\sum_{r=1}^{8} \lambda_{r}^{(n-1)} x_{r, i}=\mathcal{C}_{2 e}^{(n-1)}\left\{y_{i}\right\} .
$$

Resolvendo o sistema de equações (27), tem-se

$$
\mathbf{x}=\left[\begin{array}{cccccccc}
a_{0} & a_{1} & a_{2} & a_{3} & a_{4} & a_{5} & a_{6} & a_{7} \\
a_{0}^{*} & a_{1}^{*} & a_{2}^{*} & a_{3}^{*} & a_{4}^{*} & a_{5}^{*} & a_{6}^{*} & a_{7}^{*} \\
b_{0} & b_{1} & b_{2} & b_{3} & b_{4} & b_{5} & b_{6} & b_{7} \\
b_{0}^{*} & b_{1}^{*} & b_{2}^{*} & b_{3}^{*} & b_{4}^{*} & b_{5}^{*} & b_{6}^{*} & b_{7}^{*} \\
c_{0} & c_{1} & c_{2} & c_{3} & c_{4} & c_{5} & c_{6} & c_{7} \\
c_{0}^{*} & c_{1}^{*} & c_{2}^{*} & c_{3}^{*} & c_{4}^{*} & c_{5}^{*} & c_{6}^{*} & c_{7}^{*} \\
d_{0} & d_{1} & d_{2} & d_{3} & d_{4} & d_{5} & d_{6} & d_{7} \\
d_{0}^{*} & d_{1}^{*} & d_{2}^{*} & d_{3}^{*} & d_{4}^{*} & d_{5}^{*} & d_{6}^{*} & d_{7}^{*}
\end{array}\right] \mathbf{y}
$$

onde

$$
\begin{aligned}
\mathbf{x} & =\left[\begin{array}{lll}
x_{1, i} & \ldots & x_{8, i}
\end{array}\right]^{T}, \\
\mathbf{y} & =\left[\begin{array}{lllll}
y_{i} & \mathcal{C}_{2 e}\left\{y_{i}\right\} & \mathcal{C}_{2 e}^{2}\left\{y_{i}\right\} \ldots \mathcal{C}_{2 e}^{7}\left\{y_{i}\right\}
\end{array}\right]^{T} ;
\end{aligned}
$$

os elementos da matriz apresentada na expressão (28) são descritos na Tabela III.

De acordo com o conteúdo exposto, teoricamente, é possível o acesso simultâneo ao RAC de uma quantidade de usuários tão grande quanto se queira. Para isso, basta que se utilize 
TABELA III

ELEMENTOS DA MATRIZ-SOlUÇÃo do Sistema DE EQUAÇÕES PARA RECUPERAÇÃo DAS AUTO-SEQÜÊNCIAS DOS USUÁRIOS NO 8-RAC.

\begin{tabular}{|c|c|c|c|c|}
\hline$s$ & $a_{s}$ & $b_{s}$ & $c_{s}$ & $d_{s}$ \\
\hline \hline 0 & $-1,4294-j 0,6442$ & $1,6785-j 4,8120$ & $-1,4889+j 0,7022$ & $1,7397-j 5,2953$ \\
1 & $-1,5654-j 0,2169$ & $2,0907-j 4,6916$ & $1,6141-j 0,2572$ & $-2,1394+j 5,1042$ \\
2 & $3,6733+j 2,5955$ & $-3,6715-j 13,1277$ & $3,8180-j 2,7699$ & $-3,8198+j 14,4333$ \\
3 & $4,2796+j 1,4546$ & $-4,7862-j 12,8388$ & $-4,4162+j 1,5936$ & $4,9228-j 14,0167$ \\
4 & $-3,1118-j 3,2559$ & $3,1105+j 13,2891$ & $-3,2246+j 3,4344$ & $3,2259-j 14,5614$ \\
5 & $-3,9178-j 2,2420$ & $4,2289+j 13,0123$ & $4,0400-j 2,4010$ & $-4,3511+j 14,2218$ \\
6 & $0,8523+j 1,3240$ & $-0,8520-j 5,0445$ & $0,8797-j 1,3836$ & $-0,8800+j 5,4856$ \\
7 & $1,1926+j 1,0267$ & $-1,2731-j 4,9535$ & $-1,2284+j 1,0855$ & $1,3088-j 5,3957$ \\
\hline
\end{tabular}

uma DTT a qual esteja associado o número de autovalores necessário para definir um sistema análogo aos que foram desenvolvidos nos exemplos. No entanto, num cenário prático, algumas restrições precisariam ser consideradas.

Nesse sentido, um primeiro aspecto a ser comentado diz respeito ao esforço computacional necessário à implementação da técnica proposta. Conforme apresentado nos exemplos, a separação das seqüências dos usuários requer adições e multiplicações complexas. Dessa forma, uma avaliação do impacto dessas operações sobre o custo e a velocidade de um sistema real será de fundamental importância em trabalhos futuros.

Outro aspecto a ser considerado está relacionado à energia máxima permitida no canal de comunicação através do qual a soma das seqüências dos usuários seria transmitida. A fim de respeitar tal limitação, antes da transmissão, seria preciso multiplicar cada seqüência por um fator de escala [13]. Considerando um canal de comunicação digital, ainda se deveria avaliar detalhes relacionados à quantização dos sinais.

\section{CONCLUSÕES}

Este artigo discutiu a auto-estrutura das transformadas trigonométricas discretas. Com base na teoria desenvolvida, foi apresentado um método de múltiplo acesso ao canal somador real, onde cada usuário transmite sua informação através de auto-seqüências de uma DTT. Diversos exemplos foram apresentados, tendo sido abordados alguns aspectos para o desenvolvimento do esquema proposto.

Fatores de caráter prático dessa aplicação, como o desempenho num canal ruidoso, têm sido investigados. Além disso, têm sido estudadas as auto-estruturas de outras transformadas discretas, incluindo as definidas sobre corpos finitos, que também sirvam de base para o múltiplo acesso ao RAC.

\section{AGRADECIMENTOS}

Os autores agradecem ao Prof. Dr. Hélio Magalhães de Oliveira pelas valiosas sugestões.

\section{REFERÊNCIAS}

[1] W. Pennebaker and B. Mitchell, JPEG Still Image Data Compression Standard. Van Nostrand Reinhold, 1993.
[2] M. A. Suhail and M. S. Obaidat, "Digital Watermarking-Based DCT and JPEG Model", IEEE Trans. on Instrumentation and Measurement, v. 52, p. 1640-1647, Outubro 2003.

[3] S. A. Martucci, "Symmetric Convolution and the Discrete Sine and Cosine Transforms", IEEE Trans. on Signal Processing, vol. 42, p. 10381051, Maio 1994.

[4] R. Merched, "On OFDM and Single-Carrier Frequency-Domain Systems Based on Trigonometric Transforms", IEEE Signal Processing Letters, v. 13, p. 473-476, Agosto 2006.

[5] G. Cariolaro, T. Erseghe and P. Kraniauskas, "The Fractional Discrete Cosine Transform”, IEEE Transactions on Signal Processing, v. 50, p. 902-911, Abril 2002.

[6] G. Codevico, G. Heinig and M. Van Barel, "A Superfast Solver for Real Symmetric Toeplitz Systems Using Real Trigonometric Transformations", Numerical Linear Algebra with Applications, v. 12, N. 8, p. 699-713, 2005.

[7] J. H. McClellan and T. W. Parks, "Eigenvector Decomposition of the Discrete Fourier Transform", IEEE Transactions on Audio and Electroacoustics, v. AU-20, p. 66-74, Março 1972.

[8] S.-C. Pei and M.-H. Yeh, "The Discrete Fractional Cosine and Sine Transforms", IEEE Transactions on Signal Processing, v. 49, p. 11981207, Junho 2001

[9] C.-C. Tseng, "Eigenvalues and Eigenvectors of Generalized DFT, Generalized DHT, DCT-IV and DST-IV Matrices", IEEE Transactions on Signal Processing, v. 50, p. 866-877, Abril 2002.

[10] V. C. da Rocha Jr. and J. L. Massey, "A New Approach to the Design of Codes for the Binary Adder Channel", in Cryptography and Coding III (Ed. M. J. Ganley), IMA Conf. Series, New Series, N. 45, p. 179-185. Oxford: Claredon Press, 1993.

[11] J. Abdeljaoued and G. I. Malaschonok, "Efficient Algorithms for Computing the Characteristic Polynomial in a Domain", Journal of Pure and Applied Algebra, v. 156, N. 2-3, p. 127-145, Fevereiro 2001.

[12] J. Konvalina and V. Matache, "Palindrome Polynomials with Roots on the Unit Circle", C, R, Math. Acad. Sci. Soc. R. Can., v. 26, N. 2, p. 39-44, 2004.

[13] R. M. Campello de Souza and H. M. de Oliveira, "Eigensequences for Multiuser Communication over the Real Adder Channel", in VI International Telecommunications Symposium (ITS'2006), Fortaleza, Brasil, 2006.

[14] J. D. Gibson, The Communications Handbook. CRC Press, 2002. 